\title{
Illumination Invariant Face Image Representation Using Quaternions
}

\author{
Dayron Rizo-Rodríguez, Heydi Méndez-Vázquez, and Edel García-Reyes
}

Advanced Technologies Application Center. 7th Avenue \#21812

b/ 218 and 222, Siboney, Playa, P.C. 12200, Havana, Cuba

$\{$ drizo,hmendez, egarcia\}@cenatav.co.cu

\begin{abstract}
Variations in illumination is a well-known affecting factor of face recognition system performance. Feature extraction is one of the principal steps on a face recognition framework, where it is possible to alleviate the illumination effects on face images. The aim of this work is to study the illumination invariant properties of a hypercomplex image representation. A quaternion description from the image is built using second order derivatives decomposition. This representation is transformed to quaternion frequency domain in order to analyze its illumination invariant and discriminative properties, which are compared against the ones of the complex frequency domain representation obtained by using first order derivative decomposition. The hypercomplex quaternion representation was found to be more discriminative than the complex one, when comparing on face recognition with images under varying lighting conditions.
\end{abstract}

\section{Introduction}

Illumination invariant features are desirable in face recognition systems due to the degradation of the performance when face images are affected by lighting variations. Despite the work of Chen et al. [1], which states that no discriminative illumination invariant exists, different illumination invariant face image descriptors have been proposed. Their demonstration is based on the construction of an arbitrary object, which is able to generate the two images at hand under two different lighting sources, in a way that there is no guarantee that such images correspond to the same object or to different objects (the real and the generated one). However, when working on face image domain, faces are always detected first, which guarantees that the object which invalidates the discriminative invariant function, will no belong to the specific domain.

Some of the illumination invariant descriptors proposed for face recognition are based on statistical tools such as Principal Component Analysis and Linear Discriminant Analysis [2]. Others, transform the image to frequency domain, where illumination variations are supposed to be mainly in the low frequency spectrum, and aim at removing this frequency components while emphasizing high frequencies using them for comparison in that domain [3 5$]$. Another group of methods obtains features derived from face surface, like borders and texture

I. Bloch and R.M. Cesar, Jr. (Eds.): CIARP 2010, LNCS 6419, pp. 434 441, 2010.

(C) Springer-Verlag Berlin Heidelberg 2010 
descriptors [6, 7], taking into account morphological characteristics of the face. Among these groups of methods, the ones which work on frequency domain have shown better performance when dealing with illumination problems [8].

An image representation in the frequency domain can be described using complex components. In [9] , an initial complex description $g(x, y)$ is obtained using as components the gradient of the image $I$ in $x$ and $y$ directions, i.e.:

$$
g(x, y)=\nabla_{x} I(x, y)+\nabla_{y} I(x, y) \mathbf{j}
$$

The aim of the authors is to transform the initial representation in Eq1 to frequency domain applying Discrete Fourier Transform (DFT) and, working from cartesian and polar coordinates representation, to explore the illumination invariant properties of these four descriptions individually. The real part of cartesian coordinates yielded the best performance among them.

Hypercomplex numbers algebra has been recently used to solve pattern recognition problems. An attempt to include the quaternion representation on face recognition approaches is proposed in [10]. The authors used a two-level wavelet decomposition to obtain the image description and designed a quaternion correlation filter. They achieved very good results on CMU-PIE Database [11], but there is not any justification about quaternion usage and it seems a little empirical. Precisely, the motivation of our work is to understand and explain the reason for the inclusion of these hypercomplex numbers on face recognition frameworks.

Since it is possible to encapsulate until four bands of information in the quaternion representation, in this paper we proposed the construction of a quaternion representation using second order derivatives of the image decomposition. The proposed image representation is based on the idea in [9] but trying to obtain a more illumination invariant representation, presuming that with a wider decomposition of the image information, the lighting effects might be less perceptible. The obtained image descriptor is transformed to quaternion frequency domain and its representation in both cartesian and polar coordinates are used to compare their illumination invariant and discriminative properties among them and against those presented in [9]. Results on face image verification and identification experiments on XM2VTS and Extended Yale B databases, show that the quaternion outperforms the complex representation.

The paper is organized as follows. In Section 2, the quaternion theory and its uses in image domain is reviewed. In Section 3, face image decomposition using second order derivatives to construct the quaternion frequency domain representation is presented. The experimental results are drawn in Section 4. Finally, Section 5 gives the conclusions of the paper.

\section{Quaternion Theory}

Quaternion algebra was originally introduced by Hamilton in 1843 [12]. A quaternion is composed by a real part and an imaginary part consisting of three orthogonal components. 
The cartesian representation of quaternion numbers would be as follows.

$$
q=a+b \mathbf{i}+c \mathbf{j}+d \mathbf{k}
$$

where $a, b, c, d$ are real and $\mathbf{i}, \mathbf{j}, \mathbf{k}$ are imaginary operators.

Any quaternion may be represented in the classic polar form [13]:

$$
q=|q| e^{\mu \theta}
$$

where $\theta$ is a real angle, as the generalization of the complex exponential $e^{i \theta}=$ $\cos \theta+i \sin \theta$ by replacing $i$ by any unit pure quaternion $\mu$.

In [13] it is shown that every quaternion admits the following polar form:

$$
q=A e^{B j}
$$

where $A=a+b i$ and $B=c+d i$ are complex, and is remarked its analogy to the Cayley-Dickson form of a quaternion $q=(w+x \mathbf{i})+(y+z \mathbf{i}) \mathbf{j}=w+x \mathbf{i}+y \mathbf{j}+z \mathbf{k}$, also based on two complex numbers.

\subsection{Image Applications}

Quaternions have been used in color image processing, aiming at taking into account the true nature of vector signals [14]. According to [15], the opportunity to encode the image color information using algebraical representations provides the theoretical tools of the algebra to work with less dimensional data.

In [15], quaternion space is used to define frequency filters in color images. The author also reported the use of the frequency information of the quaternion spectrum on color image watermarking and to compute color image correlation. Color image edge detection was developed applying quaternion algebra in [16].

On the other hand, Bayro in [17] shows the improvement of two-level images when representation and processing are carried out in geometric algebra. The author states that quaternions are useful to reveal the properties of an $n$ dimensional representation of $2 \mathrm{D}$ signals and that they allow to disentangle the symmetries of $2 \mathrm{D}$ signals. A quaternion Gabor filter is designed based on this idea. The important conclusion that quaternions constitute a wide open area for the design and implementation of filters and estimators for the analysis of signals in the quaternion frequency domain, is presented.

\section{Image Quaternion Representation in the Frequency Domain}

To obtain image quaternion representation in the frequency domain, the first step is to decompose the image in four bands of information in order to use them for constructing the initial quaternion description.

We will use the second order derivatives of the face image to form the initial quaternion number, in the following way:

$$
q(x, y)=\nabla_{x x}^{2} I(x, y)+\nabla_{x y}^{2} I(x, y) \mathbf{i}+\nabla_{y x}^{2} I(x, y) \mathbf{j}+\nabla_{y y}^{2} I(x, y) \mathbf{k}
$$


where $\nabla_{x x}^{2}, \nabla_{x y}^{2}, \nabla_{y x}^{2}$ and $\nabla_{y y}^{2}$ are second order derivatives of the image. In Figure 1] a) this decomposition from a face image is depicted. This representation allows us to encode more information about image in order to find an image description in the frequency domain with better illumination invariant attributes.

Once we have the initial quaternion description, Quaternion Discrete Fourier Transform (QDFT) [14], is used to transform it to the frequency domain. The QDFT is defined as:

$$
Q(p, s)=\sum_{m=0}^{M-1} \sum_{n=0}^{N-1} e^{-\mu 2 \pi((p m / M)+(s n / M))} q(m, n)
$$

where $\mu$ is any unit pure quaternion and $q$ is the initial quaternion number obtained in Eq. (5).

After obtaining a quaternion frequency domain representation of a face image using Eq. (6) , it is possible to express it in polar coordinates by Eq. (4). We have then 8 descriptions of the image in order to analyze their illumination invariant properties and to determine the one less affected by illumination variations.

In Figure 1 (b) both cartesian and polar quaternion frequency domain components are shown. They are obtained using an initial quaternion description with the second order derivatives of the face image shown in (a).

(a)
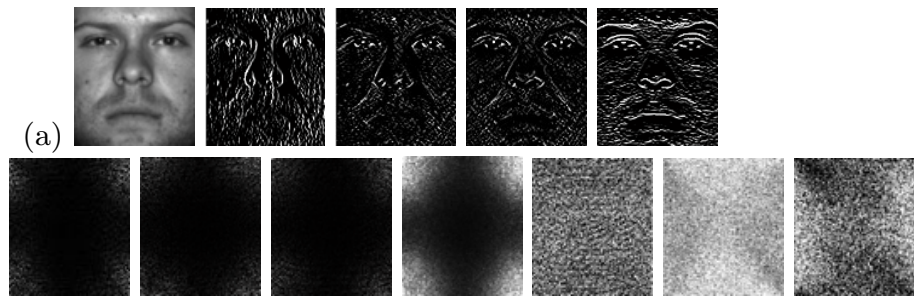

Fig. 1. Representation of (a) a face image with its second order derivatives, and (b) the quaternion frequency domain components obtained using them

\section{Experimental Evaluation}

Each quaternion frequency component is analyzed in order to determine the most illumination invariant one when used as face features and is compared with the ones obtained using the complex representation in [9]. The most invariant components that were selected, are used as face image descriptor for verification and identification experiments. Since we are less concerned with the classification step and more with the feature extraction, a simple normalized correlation is used as a similarity measure in all cases with a nearest neighbourhood classifier in the case of identification.

To analyze the different quaternion components the experimental setup described in [9] is used. The intra-class variations are analyzed comparing images 
in XM2VTS [18] database. Images corresponding to the same subject with different illumination conditions are compared among them in two experiments: in the first one (1), frontal illuminated images are compared, while in the second one (2), non frontal illuminated images are used. Table 1 shows the mean correlations obtained when comparing face images using the different components for both, the quaternion and the complex representations.

Table 1. Mean correlations obtained with quaternion and complex components

\begin{tabular}{|c|c|c|c|c||c|c|c|c|c|c|c|c|}
\cline { 2 - 11 } \multicolumn{1}{c|}{ Complex [9] } & \multicolumn{7}{c|}{ Quaternion } \\
\cline { 2 - 11 } & Real & Im & Mg & Ph & Real & Im1 & Im2 & Im3 & Polar1 & Polar2 & Polar3 & Polar4 \\
\hline \hline $\mathbf{1 )}$ & $\mathbf{0 . 8 3}$ & 0.57 & 0.69 & 0.12 & 0.50 & 0.44 & 0.46 & 0.58 & $\mathbf{0 . 8 5}$ & 0.19 & 0.25 & 0.25 \\
\hline $\mathbf{2}$ & $\mathbf{0 . 9 0}$ & 0.75 & 0.72 & 0.13 & 0.56 & 0.50 & 0.51 & 0.61 & $\mathbf{0 . 8 6}$ & 0.29 & 0.34 & 0.40 \\
\hline
\end{tabular}

It can be appreciated that Polar1 exhibits the best illumination invariant properties among quaternion components, although when comparing with the complex representation, the Real component shows a little better performance. However, this is not enough for a face recognition task, it is necessary to evaluate the discriminative properties of both descriptions in face verification and identification experiments.

\subsection{Verification Experiment}

The XM2VTS database with the Lausanne protocol [18], specifically the Configuration I, is used to verify the performance of the proposed quaternion representation in face verification setup. The XM2VTS database contains 2360 images of 295 subjects, captured in 4 different sessions. The database is divided into a Training, an evaluation (Eval) and a Test sets, each of them composed of face images under controlled illumination conditions used as clients and others as imposters. There is an additional set (Dark) which contains non frontal illuminated images from the same subjects which are also used as client and impostor comparisons.

The Equal Error Rate (EER) is the point at which the False Rejection Rate (FRR) is equal to the False Acceptance Rate (FAR). The value obtained by the classification method at this point in the Eval set is used as a threshold for the decision of acceptance or rejection in the Test and Dark sets. On the other hand, the Total Error Rate (TER) is the sum of FRR and FAR and is used to measure the performance of the verification system.

The TER obtained in each set of the database using the proposed quaternion and the complex representations is shown in Table 2. The Table also shows the results when the original face images are compared.

It can be concluded from the table, that quaternion discriminative properties are very similar to the ones of original images when they are not affected by 
Table 2. Obtained Results (TER) in Verification Experiment

\begin{tabular}{|c|c|c|c|}
\hline & Eval & Test & Dark \\
\hline Original & 0.2324 & 0.1982 & 0.9022 \\
\hline Complex & 1.0034 & 1.0007 & 1.0470 \\
\hline Quaternion & 0.3367 & 0.2924 & 0.5148 \\
\hline
\end{tabular}

illumination variations (Eval and Test sets). However, the Polar1 component of quaternion is the most discriminative feature when they are affected by illumination variations (Dark set). As can be appreciated, although the Real component of complex representation seemed to be more illumination invariant, the quaternion description is significantly more discriminative for face verification.

\subsection{Identification Experiment}

The Extended Yale B [19] database was used for face identification experiments. It contains images of 28 subjects seen under 64 different illumination conditions, in which the angle between the light source direction and the camera axis was changed each time, in a way that the larger the angle, the more unfavorable the lighting conditions are. Images with frontal angles were used as gallery and the others, were divided into 5 subsets according to the angle in the following way: S1 contains 225 images with angles between $0-12^{0}$, S2 is composed by 456 images with $13^{0}-25^{0}$ angles, S3 have 525 images with angles between $26^{0}-50^{0}$, 456 images with angles between $51^{0}-70^{\circ}$ are in S4 and S5 contains 562 images with angles between $71^{0}-130^{0}$.

Table 3. Recognition Rates (\%) obtained in Identification Experiment

\begin{tabular}{|c|c|c|c|c|c|}
\hline & S1 & S2 & S3 & S4 & S5 \\
\hline Original & 100.0 & 96.93 & 46.10 & 11.40 & 3.91 \\
\hline Complex & 100.0 & 95.39 & 37.14 & 11.40 & 3.02 \\
\hline Quaternion & 100.0 & 100.0 & 93.14 & 38.60 & 6.05 \\
\hline
\end{tabular}

The recognition rates obtained using the three different representations in each subset are presented in Table 3. Also in Figure 2 the cumulative match score vs. rank curve illustrates the performance of the three representations in S5, the most difficult one.

It can be appreciated from the Table 3 and Figure 2 that the quaternion representation outperforms both, the original and the complex representations in all subsets of the database, being more significant in those cases where the images have greater angles of variations in illumination. This also confirms the more discriminative properties of Polar1 component of quaternion representation. 


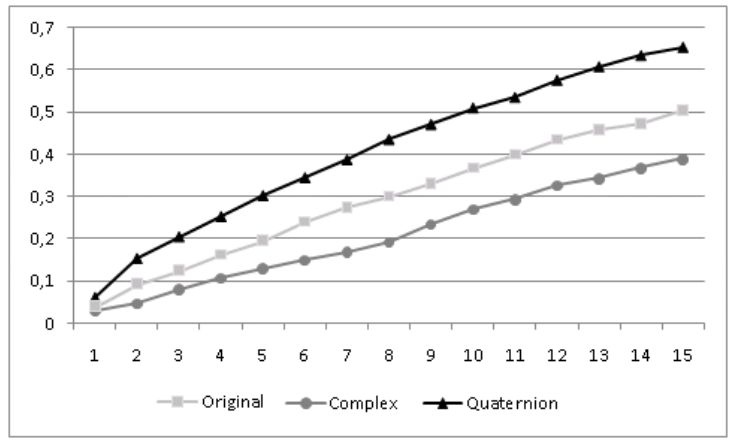

Fig. 2. S5 Cumulative Score vs. Rank Curve

\section{Conclusions and Future Works}

In this work, a quaternion representation starting from second order derivatives of face images is proposed. This representation is based on quaternion algebra properties, which encodes image information in a vectorial way. Using this representation eight quaternion components were obtained. Among them, Polar1 component proved to be the most illumination invariant one.

Although Polar1 component probed to be a feature with enough illumination invariant properties, these were a bit lower than the Real component of complex representation. Discriminative properties of both descriptions were analyzed on face recognition tasks. Results on face images verification and identification experiments confirmed that this quaternion representation is better than original images and complex representations, when dealing with images under varying lighting conditions. This shows the importance of the combination of illumination invariant and discriminative properties on face recognition frameworks.

Analyzing our work, it is possible to consider the quaternion representation as a wide area of research to develop new approaches in quaternion frequency domain. Taking into account that in complex frequency domain many face recognition methods with good performances have been developed, it can be presumed that turning to a higher dimension space as quaternions, the performance will increase. This higher dimension representation does not imply a dimensionality problem due to its algebraical properties.

As future work, it is necessary to evaluate the proposed quaternion representation with a more sophisticated similarity measure, instead of using the simple normalized correlation, which should improve the obtained results.

\section{References}

1. Chen, H., Belhumeur, P., Jacobs, D.: In search of illumination invariants. In: IEEE International Conference on Computer Vision and Pattern Recognition (2000)

2. Belhumeur, P., Pentland, J., Kriegman, D.: Eigenfaces vs. fisherfaces: Recognition using class specific linear projection. IEEE Trans. Pattern Anal. Machine Intell. 19, 711-720 (1997) 
3. Hafed, Z.M., Levine, M.D.: Face recognition using the discrete cosine transform. Int. J. Comput. Vision 43(3), 167-188 (2001)

4. Savvides, M., Vijaya Kumar, B., Khosla, P.: Corefaces-robust shift invariant pca based correlation filter for illumination tolerant face recognition. In: Proc. IEEE CVPR 2004 (2004)

5. Qing, L., Shan, S., Chen, X., Gao, W.: Face recognition under varying lighting based on the probabilistic model of gabor phase. IEEE Computer Society, Los Alamitos (2006)

6. Pujol, A.: Contributions to Shape and Texture Face Similarity Measurement. PhD thesis, Universitat Autónoma de Barcelona (2001)

7. Ahonen, T., Hadid, A., Pietikäinen, M.: Face recognition with local binary patterns. In: Pajdla, T., Matas, J(G.) (eds.) ECCV 2004. LNCS, vol. 3021, pp. 469 481. Springer, Heidelberg (2004)

8. Vijayakumar, B., Savvides, K., Venkataramani, K., Xie, C.: Spatial frequency domain image processing for biometric recognition. In: IEEE International Conference on Image Processing, pp. 53-56 (2002)

9. Garea, E., Kittler, J., Messer, K., Mendez, H.: An illumination insensitive representation for face verification in the frequency domain. In: ICPR 2006, pp. 215-218. IEEE Computer Society, Los Alamitos (2006)

10. Chunyan, X., Savvides, M., Vijayakumar, B.: Quaternion correlation filters for face recognition in wavelet domain. In: Proc. IEEE Int. Conf. on Acoustics, Speech, and Signal Processing (2005)

11. Sim, T., Baker, S., Bsat, M.: The cmu pose, illumination, and expression (pie) database of human faces. Technical report (2007)

12. Hamilton, W.: 24. In: Elements of Quaternions. Longmans, Green (1866)

13. Sangwine, S., Le Bihan, N.: Quaternion polar representation with a complex modulus and complex argument inspired by the cayley-dickson form. In: Proc. IEEE Int. Conf. on Acoustics, Speech, and Signal Processing (2005)

14. Moxey, C., Sangwine, S., Ell, T.: Hypercomplex correlation techniques for vector images. IEEE Transaction on Signal Processing 51, 1941-1953 (2003)

15. Denis, P.: Quaternions et Algèbres Géométriques, de nouveaux outils pour les images numériques couleur. PhD thesis, Université de Poitiers (2006)

16. Mahecha, V.: Aplicación de los números hipercomplejos o cuaterniones en imágenes de color. Ingeniería y desarrollo (023), 72-83 (2008)

17. Bayro, E.: 6. In: Geometric Computing for Perception Action Systems, pp. 115136. Springer, New York (2001)

18. Messer, K., Matas, J., Kittler, J., Jonsson, K.: Xm2vtsdb: The extended m2vts database. In: Second International Conference on Audio and Video-based Biometric Person Authentication, pp. 72-77 (1999)

19. Lee, K.C., Ho, J., Kriegman, D.J.: Acquiring linear subspaces for face recognition under variable lighting. IEEE Transactions on Pattern Analysis and Machine Intelligence 27(5), 684-698 (2005) 